\title{
Carbon Nanotube Fabric Cooling System for Firefighters and First Responders: Modeling and Simulation
}

\author{
J. Sullivan ${ }^{\text {a }}$, M. Schulz ${ }^{\text {a,* }}$, K. Vemaganti ${ }^{\mathrm{a}}$, A. Bhattacharya ${ }^{\mathrm{b}}$, B. J. Jetter ${ }^{\mathrm{c}}$ \\ V. Shanov ${ }^{a}$, N. Alvarez ${ }^{a}$, Jay Kim ${ }^{\mathrm{d}}$ \\ ${ }^{a}$ Nanoworld Laboratories, Univ. of Cincinnati, Cincinnati, Ohio 45221, the U.S.A. \\ ${ }^{\mathrm{b}}$ Dept. of Environmental Health, P.O. Box 670056, 3223 Eden Avenue, Cincinnati, Ohio 45267, \\ the U.S.A. \\ ${ }^{\mathrm{c}}$ Monroe Fire Department Interim Fire Chief, Ohio 45044, the U.S.A. \\ ${ }^{\mathrm{d}}$ Mechanical and Materials Engineering, Univ. of Cincinnati, Cincinnati, Ohio 45221, the U.S.A.
}

\begin{abstract}
This study investigates carbon nanotube textiles as advanced personal protection equipment for firefighters and first responders. Carbon nanotubes are lightweight, flame resistant, and possess high mechanical and thermal properties. Carbon nanotubes are also thermally anisotropic, meaning they easily conduct heat along the axis of an individual tube, and are relatively insulating across the tube's diameter. By recognizing this anisotropic behavior, heat transfer through a layer of aligned carbon nanotubes in a garment can be partially redirected to a cold reservoir thereby protecting the wearer from heat stress and exhaustion. Finite element models were developed to simulate a carbon nanotube layer embedded in a firefighting garment and thermally connected to a cold reservoir. Simulation showed that under heat stress conditions, firefighter skin temperature was considerably reduced by the cooling layer.
\end{abstract}

Keywords: Firefighter; Personal Protection; Carbon Nanotube Textile; Cooling

\section{Introduction}

Firefighting is a dangerous profession. Heat stress and heat exhaustion decrease performance and increase the risk for injury or death for firefighters and other first responders. In 2010, 87 firefighters died on duty in the US, and $63.2 \%$ of those fatalities were from heat-related complications [1]. The materials used to protect firefighters from heat were developed over 50 years ago (Kevlar/Nomex) and rely solely on passively insulating the wearer from the outside environment. If a firefighter garment could protect firefighters from the outside environment and also internal metabolic heat by directing heat out of the garment, it could reduce heat-stress-related fatalities.

\footnotetext{
${ }^{*}$ Corresponding author.

Email address: mark.j.schulz@uc.edu (M. Schulz).
} 
Carbon nanotubes (CNTs) have extraordinary mechanical [2-4] and thermal [5-7] properties, and are also lightweight [4], flame-resistant [8], and hydrophobic [8]. CNTs also have thermally anisotropic behavior [9]; a single CNT fiber is highly conductive along its axis and relativity insulating across its diameter. These properties may enable the next generation of firefighter Personal Protective Equipment (PPE) to be developed. By creating a bulk textile material where the fibers are aligned in the plane of the material (creating a material that is conductive along one axis in-plane and insulating in the other orientations), a hypothesis can be formed: CNT textiles thermally connected to a cold reservoir or sink could improve occupational safety for firefighters and other first responders [10]. This would be achieved by redirecting the heat flux away from the wearer therefore reducing the heat stress on the wearer. Heat enters the firefighters garment due to exposure to the environment and also from heat generated by the body. In this new approach called a smart garment, heat is transferred from the garment to an external cold reservoir or sink (i.e. to a lower temperature material than the wearer and the ambient environment). The design of the smart cooled garment is illustrated in Fig. 1.

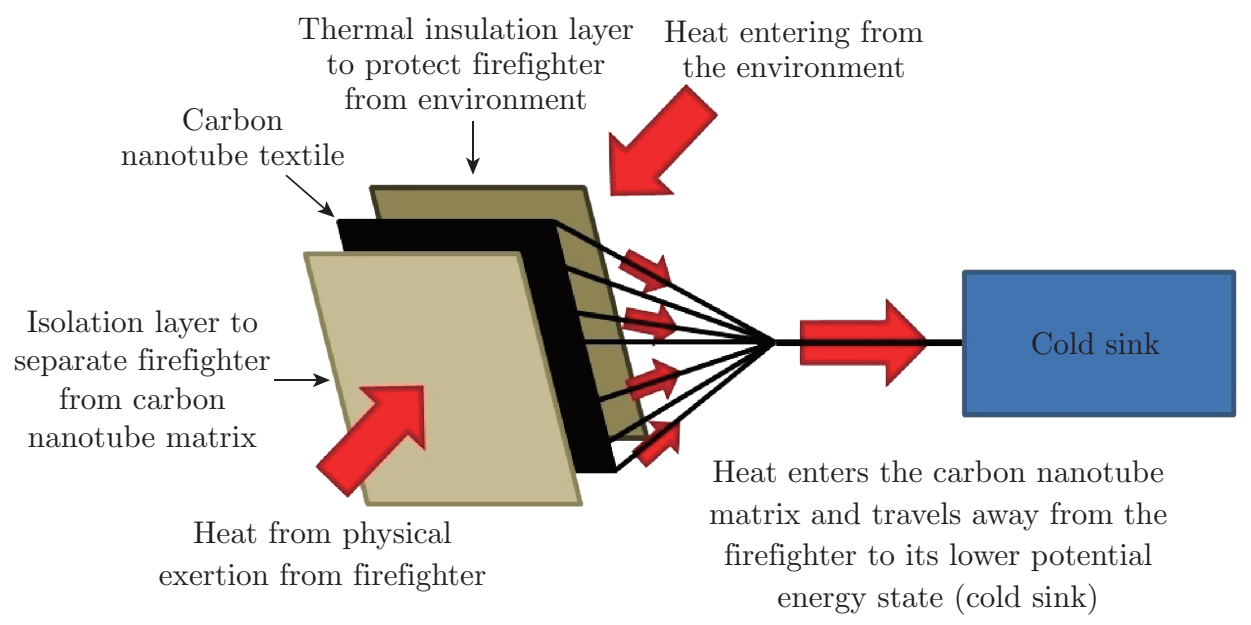

Fig. 1: Schematic of smart cooling for protecting firefighters from heat stress. The smart system draws heat out of the garment to a cold reservoir or sink rather than just relying on passive heat insulation from the environment to keep the firefighter cool

\section{Methodology}

Finite Element Analysis (FEA) models were constructed to test the effectiveness of CNT fabric integrated into firefighting garments supplemented with a cold reservoir to protect the wearer from heat stress. Modeling is a cost-effective way to rapidly evaluate the performance of nanotube material without having to manufacture test garments of different designs. This simulation based approach was chosen to give a first indication how successfully heat can be piped out of a firefighter garment. The FEA models were also generated because the parameters of the system can easily be changed to obtain a deeper understanding of the components' interactions with each other and to aid in future design. The model developed herein provides guidelines on performance of the proposed design, but is an approximate model. A more elaborate model for the firefighter and garment system would add too much complexity (environment, garment, cold reservoir, and body) at this time. Thus experimental testing of fabric swatches is the next step. Looking ahead, 
producing a prototype firefighter suit to experimentally test this method is also feasible, but the effort of prototyping must be first justified by simulation and coupon testing [11].

A FEA model is used to discretize the garment into elements to perform the thermal analysis. In this case, the heat equation is given by:

$$
\frac{\partial}{\partial x}\left(k_{x} \frac{\partial T}{\partial x}\right)+\frac{\partial}{\partial y}\left(k_{y} \frac{\partial T}{\partial y}\right)+\frac{\partial}{\partial z}\left(k_{z} \frac{\partial T}{\partial z}\right)+\dot{q}=\rho c_{p} \frac{\partial T}{\partial t}
$$

where: $T=$ Temperature, $t=$ time, $k=$ thermal conductivity, $\dot{q}=$ heat generated at the boundary, and $c_{p}=$ specific heat. Here, only conduction heat transfer is modeled. The numerical solution is found by iteratively solving the heat equations for every finite element, given the initial conditions for each element and the specified boundary conditions. The iteration is performed until the solution converges. This is done for each time step. The commercial software used for the analysis is ANSYS.

In this simulation, only the core section of the body was modeled as it houses the vital organs. To best represent the system and reduce assumptions, the body of the wearer, each individual layer of the garment, the cold reservoir, the air gap between the body and the garment, and the environment were modeled within the selected boundary. The CNT textile was modeled to be a part of the firefighting garment, surrounded by traditional fabric on either side. The cold reservoir was connected to the CNT layer in the center of the model.

The core of the body was assumed to be cylindrical so a 2D axisymmetric model could be used. The axisymmetric assumption requires that around an axis of rotation, the system, boundary conditions and solution are symmetric. The axisymmetric assumption allows the model to be a $2 \mathrm{D}$ area, which represents the $3 \mathrm{D}$ volume of the $2 \mathrm{D}$ area revolved around an axis. This greatly reduces the complexity of the model without compromising the accuracy. The mesh has a high density of elements near and around the fabric to capture the effects of small geometry, and the large thermal gradients expected in that location. The mesh becomes less dense towards the body, as there are no small geometries to capture and large thermal gradients are not expected there. The boundary conditions applied to the system represent convection from the environment, metabolic heat rate (the metabolic rate is used to estimate the heat being generated by the body [12]), and a constant temperature boundary representing the cold reservoir. Fig. 2 shows the geometry used for the model, the corresponding discretized geometry (mesh), and a schematic of the model.

A parameter study was performed for the system by subjecting the model to four different conditions: (1) Hot environment $\left(40^{\circ} \mathrm{C}\right.$ [13]) and light work (332 Watts [13]); (2) Hot environment and strenuous work (889 Watts [14]); (3) Firefighting environment $\left(58^{\circ} \mathrm{C}[15]\right)$ and light work; and (4) Firefighting environment and strenuous work. Within these four cases, parameters of the firefighter garment were also changed: two thermal conductivities of the CNT layer, two thicknesses of the CNT layer, and two temperatures for the cold reservoir were evaluated in the study. Together, thirty-two different simulations were performed. Four additional simulations were performed (one for each condition case) without the CNT layer and cold reservoir to represent the traditional garment and provide results for comparison. Creating many models with different parameters and observing the difference in the cooling effectiveness is an aid to future design of firefighter turnout gear. The model simulates twenty minutes of exposure to the environment. This was done following a study from Wang et al. which showed that the skin temperature of firefighters wearing firefighter garments in hot conditions while being physical active had the highest rate of change during the first 20 minutes of exposure [13]. 

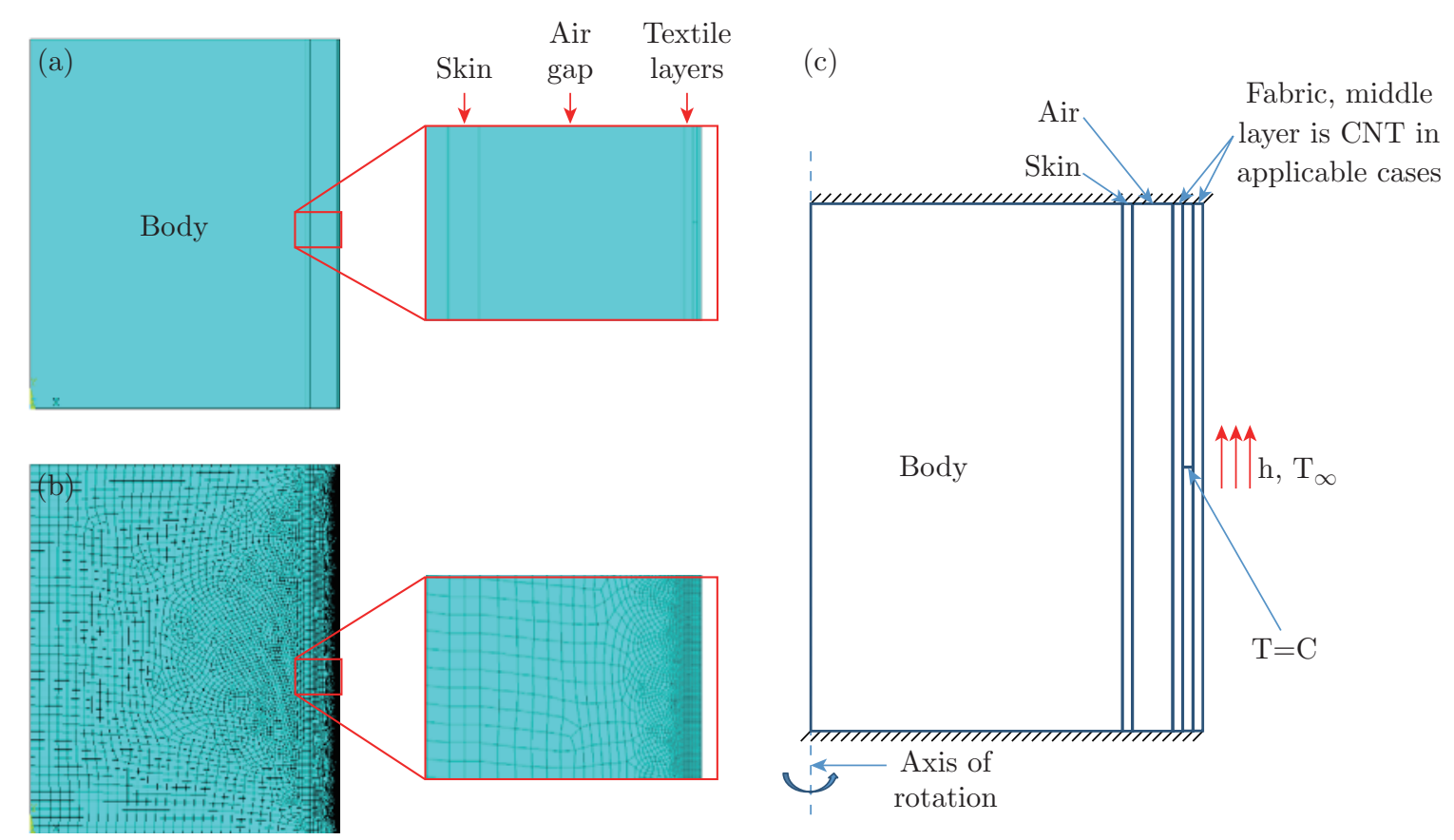

Fig. 2: Modeling of the firefighter and garment: (a) Model geometry; (b) Meshed geometry; (c) Schematic of the model; Boundary conditions are in red

Two thermal conductivities for the CNT were considered in the simulation. These were the highest thermal conductivity of CNT thread (a candidate material to produce CNT textiles) that was measured at the time of the study [4], and the highest thermal conductivity of any CNT material reported at the time of the study [16]. The latter value was used to extrapolate thermal conductivities of CNT threads that can be expected in the future. However, these reports only provide the conductivity in the axial direction. The conductivity in the transverse direction across the diameter is difficult to measure experimentally due to the small size of the material (the average diameter of CNT thread is $20 \mu \mathrm{m}$ ). The thermal conductivity in the radial direction for the simulations was estimated by taking the ratio of the thermal conductivities found by Che et al. in their molecular dynamic simulations for CNT thread. Assuming this relationship is constant, an estimated thermal conductivity in the radial direction can be found by using this ratio and experimental values for the axial thermal conductivity. The ratio was found to be $k_{\text {axial }} / k_{\text {radial }}$ $=173[9]$. The model has the CNTs oriented so that the conductive axis is aligned vertically. This creates a thermal path from the CNTs to the cold reservoir and places the less-conductive orientation of the CNTs parallel to the axis in which the heat is entering the body. The human body was modeled as plain water. Comparing the thermal properties of different body tissues to the thermal properties of water indicates this is a valid assumption [17]. The thermal properties of the materials used in the model are listed in Table 1.

The average chest circumference of a male firefighter is $1.24 \mathrm{~m}$ [24]. This value was used to find a circle with an equivalent circumference to represent the body. The radius of an average male firefighter's body was then found to be $0.2 \mathrm{~m}$. The thickness of the firefighting garment materials were measured from samples using a digital caliper; the thicknesses were 0.4, 0.4, and $0.9 \mathrm{~mm}$ for the outer shell, moisture barrier, and thermal liner, respectively (the three layers that constitute the firefighter PPE). The thickness of the air gap between the body and the fabric was $0.02 \mathrm{~m}$ [25]. The thickness of the CNT layer was $0.4 \mathrm{~mm}$, which is approximately the thickness of seven 
Table 1: Thermal properties and density of the materials used in the model

\begin{tabular}{ccccccc}
\hline $\begin{array}{c}\text { Material and } \\
\text { Properties }\end{array}$ & $\begin{array}{c}\text { CNT [4], } \\
{[16],[18]}\end{array}$ & $\begin{array}{c}\text { Outer Shell } \\
{[19-21]}\end{array}$ & $\begin{array}{c}\text { Moisture Barrier } \\
{[21],[22]}\end{array}$ & $\begin{array}{c}\text { Thermal Liner } \\
{[20],[21],[23]}\end{array}$ & $\begin{array}{c}\text { Human Body } \\
{[17]}\end{array}$ & Air [17] \\
\hline$\rho\left(\frac{k g}{m^{3}}\right)$ & 900 & 1410 & 1380 & 1416 & 1000 & 1.16 \\
$k\left(\frac{W}{m K}\right)$ & $80,262^{*}$ & 0.145 & 0.25 & 0.125 & 0.613 & 26.3 \\
$c_{p}\left(\frac{k J}{k g K}\right)$ & 0.830 & 1.338 & 1.256 & 1.354 & 4.179 & 1.007 \\
\hline
\end{tabular}

Lower and upper range axial values are listed, the corresponding approximate radial values for through-thethickness conduction are; $0.46,1.73 \frac{\mathrm{W}}{\mathrm{mK}}$, respectively.

CNT sheets overlaid. The moisture barrier was replaced by the CNT layer in the simulations with the CNT layer. The CNT layer and the moisture barrier are both approximately the same thickness, and CNTs are extremely hydrophobic, therefore fulfilling the purpose of the moisture barrier [8].

The heat transfer coefficient for the environment to the fabric was estimated using empirical equations from the ASHRAE handbook for a person on a treadmill in still air with the ambient air pressure taken into consideration [26]. Using these equations, a heat transfer coefficient of 17.9 W/m was found. Values from the study by Wang et al. were used to solve these equations [13]. The cold reservoir was tested at $0{ }^{\circ} \mathrm{C}$ (to represent ice), and $20^{\circ} \mathrm{C}$ (a point in between that of ice and the body temperature).

The initial conditions of the model were broken into four parts: (i) The inner half of the body was set to the core temperature $\left(37.64{ }^{\circ} \mathrm{C}\right.$ ); (ii) The outer half was set to the average of the core temperature and the skin temperature $\left(35.32{ }^{\circ} \mathrm{C}\right)$; (iii) The skin boundary was set to skin temperature $\left(33^{\circ} \mathrm{C}\right)$; and (iv) The air gap and the fabric layer were set to the difference between the skin and the ambient air $\left(36.5^{\circ} \mathrm{C}\right.$ for $\mathrm{T}_{\infty}=40{ }^{\circ} \mathrm{C}, 45.8{ }^{\circ} \mathrm{C}$ for $\left.\mathrm{T}_{\infty}=58.5^{\circ} \mathrm{C}\right)$.

\section{Model Validation}

To verify that the solutions obtained using the models were valid (i.e., that the solutions are independent of the mesh and time step used and the solutions are representative of actual physical phenomena), mesh and time step independence studies were performed, and the solution of a model without CNT was compared against measured data. Two additional meshes were generated for the mesh independence study (half the original mesh density, and twice the original mesh density), while leaving all other parameters the same, and the results were compared to the results from the simulation using the original mesh. For the time step independence study, two additional simulations were performed with a time step half as large as the time step used, and with a time step twice as large as the time step used. No other parameters were changed in the time step study. The final average skin temperatures for all four cases were less than a tenth of a degree Celsius different from the standard case. This shows that the solutions found are independent of the mesh and time step used.

Wang et al. exposed six subjects to a controlled hot environment while walking on a treadmill and wearing firefighting protective equipment, and measured their change in skin temperature 
over time [13]. To validate the accuracy of the model, the model was used to simulate the study by Wang et al. and the results were compared. Fig. 3 shows the temperature profile found using the model, overlaid with Wang et al.'s measurements. The coefficient of determination $\left(r^{2}\right)$ between the simulation and the measured results is 0.89 , which is a high correlation. This shows that the model matches the measured data well for this particular case.

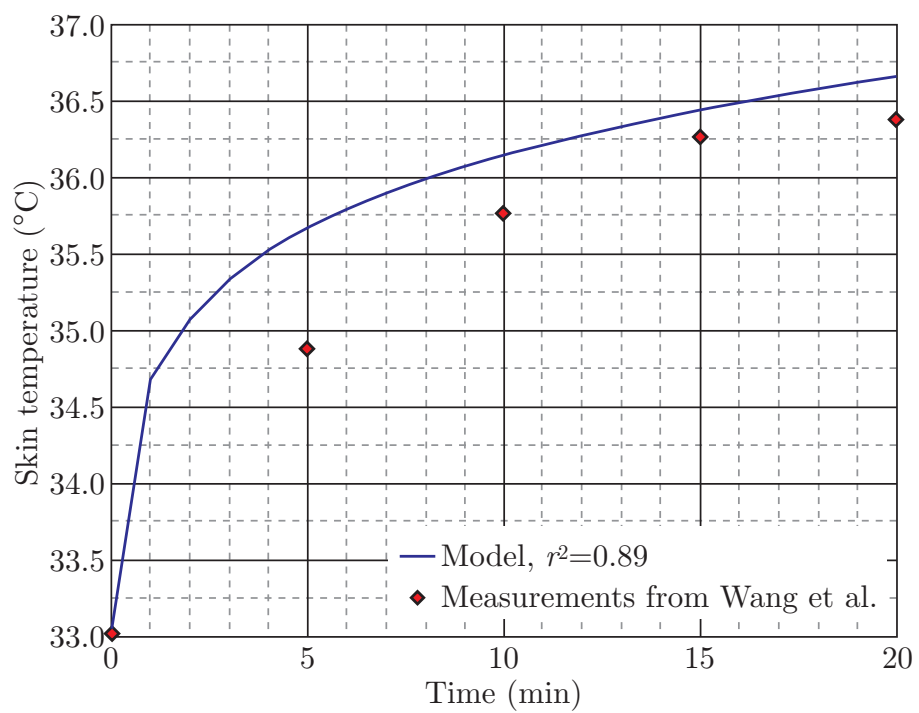

Fig. 3: Comparing skin temperature vs time from the FEM simulation to Wang et al.'s experiments [13]

\section{Simulation Results}

Table 2 shows the average skin temperature for each simulation after 20 minutes. The average skin temperatures found with the CNT and cold reservoir cases were compared to the respective datum case of the standard firefighting garment to observe the improvement using a CNT layer with a cold reservoir. It can be seen in the table that with the CNT layer and cold reservoir, the skin temperature after exposure was on average $6{ }^{\circ} \mathrm{C}$ lower than the models without the CNT layer and cold reservoir. Only half the cases are shown because the metabolic rate was determined to be insignificant compared to the convection boundary condition and the effects of the CNT and cold reservoir. To provide a quantitative interpretation to the results: cells can only survive $+42{ }^{\circ} \mathrm{C}$ temperatures for a limited time (at $50{ }^{\circ} \mathrm{C}$ cells will start to die after about $16 \mathrm{~min}$ ) [27]. The datum cases in Table 2 are for the firefighting garment with no CNT layer, i.e., the moisture barrier layer is used in place of the CNT layer.

The overall general effects of the parameters of the CNT layer and environment are: (i) The thicker CNT layer increases cooling and reduces skin temperature; (ii) The higher thermal conductivity CNT layer reduces skin temperature; (iii) The higher environmental temperature increases skin temperature; and (iv) The lower reservoir temperature produced lower skin temperature and has a greater effect than doubling the thickness of the CNT layer. These results all make physical sense and provide guidelines on future design of smart garments. It is encouraging that even with the cold reservoir at the higher temperature of $20^{\circ} \mathrm{C}$, and using the thicker CNT layer with the lower thermal conductivity (the easiest to achieve design), the skin temperature was reduced significantly, 3.7 and $5{ }^{\circ} \mathrm{C}$ for the two environmental temperatures. This indicates the 
Table 2: Results from the simulation

\begin{tabular}{|c|c|c|c|c|c|c|c|c|}
\hline \multicolumn{9}{|c|}{$T_{\infty}=40^{\circ} \mathrm{C}$} \\
\hline$T_{\text {skin,datum }}\left({ }^{\circ} \mathrm{C}\right)$ & \multicolumn{4}{|c|}{$k_{\mathrm{CNT}, \text { axial }}=80 \mathrm{~W} / \mathrm{mk}$} & \multicolumn{4}{|c|}{$k_{\mathrm{CNT}, \text { axial }}=272 \mathrm{~W} / \mathrm{mk}$} \\
\hline \multirow{2}{*}{36.9} & \multicolumn{2}{|c|}{$t_{\mathrm{CNT}}=0.4 \mathrm{~mm}$} & \multicolumn{2}{|c|}{$t_{\mathrm{CNT}}=0.2 \mathrm{~mm}$} & \multicolumn{2}{|c|}{$t_{\mathrm{CNT}}=0.4 \mathrm{~mm}$} & \multicolumn{2}{|c|}{$t_{\mathrm{CNT}}=0.2 \mathrm{~mm}$} \\
\hline & $T_{\text {sink }}=0{ }^{\circ} \mathrm{C}$ & $T_{\text {sink }}=20^{\circ} \mathrm{C}$ & $T_{\text {sink }}=0{ }^{\circ} \mathrm{C}$ & $T_{\text {sink }}=20^{\circ} \mathrm{C}$ & $T_{\text {sink }}=0^{\circ} \mathrm{C}$ & $T_{\text {sink }}=20^{\circ} \mathrm{C}$ & $T_{\text {sink }}=0{ }^{\circ} \mathrm{C}$ & $T_{\text {sink }}=20^{\circ} \mathrm{C}$ \\
\hline$T_{\text {skin,avg }}\left({ }^{\circ} \mathrm{C}\right)$ & 28.8 & 33.2 & 30.6 & 33.9 & 24.1 & 31.0 & 27.0 & 32.3 \\
\hline$\Delta T\left({ }^{\circ} \mathrm{C}\right)$ & 8.1 & 3.7 & 6.3 & 3.0 & 12.8 & 5.9 & 9.9 & 4.6 \\
\hline \multicolumn{9}{|c|}{$T_{\infty}=58{ }^{\circ} \mathrm{C}$} \\
\hline$T_{\text {skin,datum }}\left({ }^{\circ} \mathrm{C}\right)$ & \multicolumn{4}{|c|}{$k_{\mathrm{CNT}, \text { axial }}=80 \mathrm{~W} / \mathrm{mk}$} & \multicolumn{4}{|c|}{$k_{\mathrm{CNT}, \text { axial }}=272 \mathrm{~W} / \mathrm{mk}$} \\
\hline \multirow{2}{*}{42.5} & \multicolumn{2}{|c|}{$t_{\mathrm{sink}}=0.4 \mathrm{~mm}$} & \multicolumn{2}{|c|}{$t_{\mathrm{sink}}=0.2 \mathrm{~mm}$} & \multicolumn{2}{|c|}{$t_{\operatorname{sink}}=0.4 \mathrm{~mm}$} & \multicolumn{2}{|c|}{$t_{\text {sink }}=0.2 \mathrm{~mm}$} \\
\hline & $T_{\text {sink }}=0{ }^{\circ} \mathrm{C}$ & $T_{\text {sink }}=20^{\circ} \mathrm{C}$ & $T_{\text {sink }}=0{ }^{\circ} \mathrm{C}$ & $T_{\text {sink }}=20^{\circ} \mathrm{C}$ & $T_{\text {sink }}=0{ }^{\circ} \mathrm{C}$ & $T_{\text {sink }}=20^{\circ} \mathrm{C}$ & $T_{\text {sink }}=0{ }^{\circ} \mathrm{C}$ & $T_{\text {sink }}=20^{\circ} \mathrm{C}$ \\
\hline$T_{\text {skin }, \operatorname{avg}}\left({ }^{\circ} \mathrm{C}\right)$ & 33.1 & 37.5 & 35.2 & 38.6 & 27.6 & 34.6 & 31.0 & 36.3 \\
\hline$\Delta T\left({ }^{\circ} \mathrm{C}\right)$ & 9.4 & 5.0 & 7.4 & 3.9 & 15.0 & 7.9 & 11.5 & 6.2 \\
\hline
\end{tabular}

smart garment may increase safety for firefighters. The additional weight of the cooling system and cost are to be determined.

An additional simulation was conducted with the CNT layer not connected to a cold reservoir to see how this would affect the skin temperature over time. It was expected that this would be detrimental to the firefighter as even out of plane, the CNT fabric would be less insulating than traditional fabric. This was observed. The results are shown in Fig. 4.

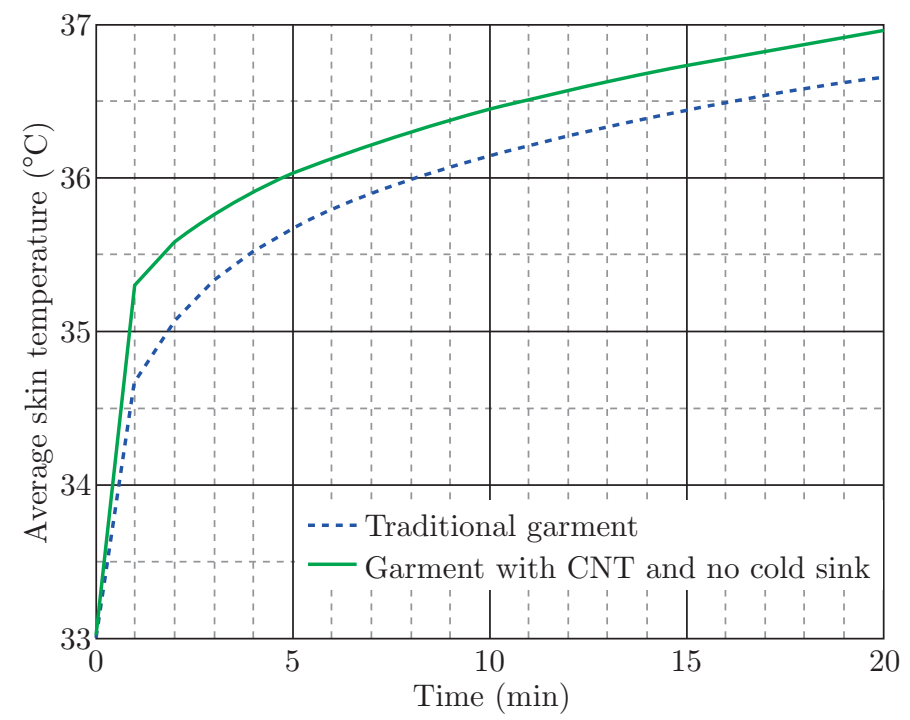

Fig. 4: Simulation with CNT and no cold reservoir compared to a simulation modeling traditional firefighter garments

The simulation results with the CNT layer but no cold reservoir in Fig. 4 are important because they show the CNT layer can increase skin temperature if the cold reservoir fails. This is because the CNT layer has greater transverse thermal conductivity than the moisture barrier layer (the CNT layer replaces the moisture barrier when the CNT layer is used). Thus a suggestion to make future designs fail-safe, is to add the CNT layer in addition to the moisture layer (not replacing 
the moisture layer).

\section{Discussion}

Every simulation with the CNT layer and cold reservoir showed that, after exposure, the average skin temperature of the firefighter was significantly reduced from the corresponding case without the CNT layer and cold reservoir. On average, the average skin temperature after exposure was $6^{\circ} \mathrm{C}$ lower for the models with the CNT layer and cold reservoir than the models without the CNT layer and cold reservoir. In the cases where the ambient temperature was set to firefighting temperatures, it was found that with only the traditional passive firefighter garments for protection, the wearer would start to be endangered by the exposure. Comparing this to the corresponding models with the CNT layer and cold reservoir, the average reservoir temperatures are only slightly elevated after exposure. Some of the CNT and cold c cases cooled the skin below the comfort zone to a point that may cause peripheral vasoconstriction, which could create a new hazard for the firefighters [28]. It has been shown for liquid cooled garments (where a refrigeration cycle and network of tubing is used to regulate the wearer's body temperature) that if the cooling system is allowed to cycle on and off, overcooling can be mitigated [28]. The simulation in the future should consider a mechanism to regulate the cooling to prevent overcooling. The simulation model also does not account for the thermal storage of the cold reservoir. The model assumes that the cold reservoir has an infinite amount of thermal storage. In actual implementation, work needs to be done to the system (such as a the refrigeration cycle in the liquid cooled garments), or an endothermic chemical reaction needs to be applied to the cold reservoir so the heat can be removed at a more constant rate otherwise the cold reservoir will heat up and lose the ability to store energy. If ice was used to cool the cold reservoir, after the ice loses its latent heat due to phase change, the cold reservoir will heat up over time. A more elaborate model would be needed to incorporate the cold reservoir's thermal storage.

This model does not include mass transport. This means that a major mode in which heat is transferred within the body (blood flow), and a major mode for the body to remove heat (sweat) are not accounted for [12]. Additionally, this model does not account for fluid flow through or around the fabric (air or sweat). The effect of these simplifications appears to be minor, as the model had a high correlation to measured data. This suggests that the model is accurate enough for an initial assessment of the feasibility of adding a CNT layer and cold reservoir addition to firefighter's PPE. A better model of the system could be generated by using more elaborate governing equations that include terms to approximate the effect of these modes of heat transfer, without having to make very detailed geometries or run very complicated simulations [29, 30]. This would allow the core body temperature to be reported, which would reveal more of the effects of heat on the firefighter than just skin temperature.

After this study was finished, new reports of CNT threads with higher thermal conductivities were reported. Behabtu et al. reported that the thermal conductivity of their CNT thread is $380 \mathrm{~W} / \mathrm{mK}$ as spun and $635 \mathrm{~W} / \mathrm{mK}$ after doping [7]. Behabtu et al. reported that their material is significantly more conductive than the previous reports, where the material properties were referenced for these simulations. The firefighter simulations showed that increased thermal conductivity significantly increased the effectiveness of the system, so it can be extrapolated that using these new heat transfer values, the simulations would show even better effectiveness, assuming the high quality CNT fabric can be produced. 
The main take-away from this paper is that the thermal conductivity of nanotube layer is so high that it draws the heat from the garment and almost keeps the skin temperature at the initial value of $33{ }^{\circ} \mathrm{C}$ even though the firefighter is in a hot environment fighting the fire. In some cases, the CNT layer cooling system overcooled the firefighter implying that some type of thermostatic control may be needed for the smart garment. Going further, there are other interesting applications of the smart garment with the CNT layer and cooling system [32, 33]. First responders and any workers that need cooling can use the smart garment. In athletic apparel, the CNT fabric would conduct heat from the athlete to the cooler air environment, and a separate cold reservoir may not be needed. On the other hand, a winter coat made of CNT fabric would not be a good idea as the body heat would be drawn out into the cold air. In structural applications, e.g., in aircraft hot sections near the engines, a CNT layer could draw heat to cooler zones in the structure or into the air. In general, a CNT layer could be used wherever high heat conduction is needed. Additional characteristics are the CNT layer is electrically conductive, can act as a shield for electromagnetic interference, it is strong, abrasion resistant, can be chemically functionalized to neutralize potentially toxic substances, it may shield certain types of radiation, it is the blackest fabric known to man and thus will highly absorb heat from sunlight, and the surface area of the fabric will provide high evaporation or if functionalized, high absorption of water. Safety and cost are the two main impediments to applications of CNT textiles. We must be sure the nanotubes are not released from the fabric, and the manufacturing method must be scalable. Longer nanotubes and post processing are being pursued to reduce the likelihood of nanotubes being released from the fabric. A new reactor design that directly produces nanotube sheet is in the research study phase and could continuously produce CNT sheet at lower cost. Thus we expect that nanotube smart textiles will set a new benchmark in design and performance of personal protective clothing and multifunctional composite materials.

\subsection{Future Work}

Future work planned is to fabricate a carbon nanotube sheet layer and test it for thermal conductivity [31]. Then the layer can be integrated into a firefighter garment and tested using a firefighter mannequin. Fig. 5 shows early production of a CNT sheet with the fibers aligned in the plane of the sheet (as the CNT material in the FEA models). In the future, samples of this material will be integrated into firefighter turnout gear and used to test the cooling effectiveness of a smart garment by measuring the amount of heat flux that can be redirected by the nanotube sheet layer.

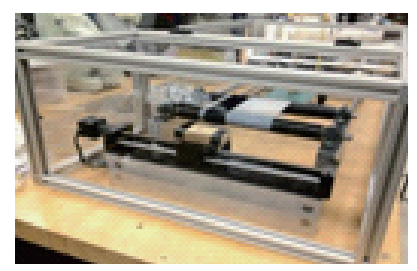

(a)

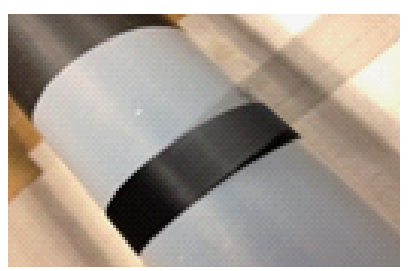

(b)

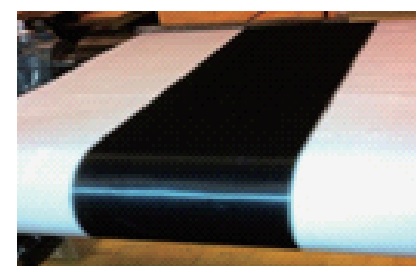

(c)

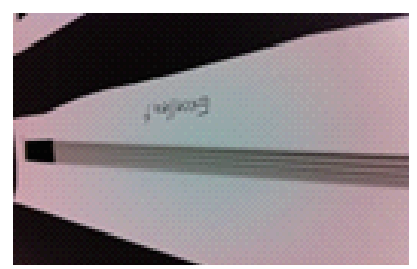

(d)

Fig. 5: Manufacturing CNT sheet in the UC Nanoworld Lab [32]: (a) Machine for drawing a CNT forest from a wafer on a moveable carriage to form a sheet on a rotating belt; (b) Close-up view of the nanotube ribbon being wound onto a drum; (c) The nanotube sheet on the belt; (d) The sheet cut and unwrapped from the belt, a long ribbon is shown for reference, 100 or more layers or ribbon form the sheet 


\section{Summary and Conclusions}

This work has made progress in evaluating the viability of using CNT textiles to increase the occupational safety for firefighters and other first responders. The proposed CNT layer and cold reservoir is a simpler approach for cooling garments than alternative liquid-cooled garments. Both systems perform the same task, yet the CNT cold reservoir method adds modest extra weight, but no extra bulky equipment such as pumps, tubing, and batteries, while having potential benefits of increasing the abrasion resistance, flame resistance, etc of the garment. The next steps in the development of the firefighter smart garment are: making samples of the CNT fabric and measuring the thermal conductivity in all directions, updating the model and simulation using the measured thermal conductivity values, obtaining a preliminary design for the cold reservoir and fabric, testing the fabric and cold reservoir on the bench, and then building a garment with a smart layer and testing. Conclusions are that the CNT layer should be an additional layer in firefighter garments, the garment design should be fail safe if the cold reservoir is exhausted, and there are other possible applications for a CNT fabric layer including for athletic apparel.

\section{Acknowledgment}

This project is supported by the NIOSH Educational and Research Center grant. Assistance on this project was provided by students Anli Wang and Jason Sookoor, Professor Tina Reponen, Dr. Gary Conroy, and Mr. Andrew Schwartz of Lion Products Company. The nanotube research aspect of the paper was supported by the NSF grant SNM GOALI: Carbon Nanotube Superfiber to Revolutionize Engineering Designs, Award: 1120382.

\section{References}

[1] Firefighter Fatalities in the United States in 2010, U. S. Department of Homeland Security, Federal Emergency Management Agency (FEMA), U. S. Fire Administration, National Fire Data Center and The National Fallen Firefighters Foundation, 500 C Street SW, Washington, DC 20472, 2011

[2] B. Demczyk, Y. Wang, J. Cumings, M. Hetman, W. Han, A. Zettl, R. Ritchie, Direct mechanical measurement of the tensile strength and elastic modulus of multiwalled carbon nanotubes, Mater. Sci. Eng. A, 2002, 334(1-2), 173-178

[3] Q. Lu, G. Keskar, R. Ciocan, R. Rao, R. Mathur, A. Rao, L. Larcom, Determination of carbon nanotube density by gradient sedimentation, J. Phys. Chem. B, 2006, 110(48), 24371-24376

[4] M. Jakubinek, M. Johnson, M. White, C. Jayasinghe, G. Li, W. Cho, M. J. Schulz, V. N. Shanov, Thermal and electrical conductivity of array-spun multi-walled carbon nanotube yarns, Carbon N. Y., 2012, 50(1), 244-248

[5] P. Kim, L. Shi, A. Majumdar, P. McEuen, Thermal Transport Measurements of Individual Multiwalled Nanotubes, Phys. Rev. Lett., 2001, 87(21), 215502, 1-4

[6] J. Hone, M. Llaguno, M. Biercuk, A. Johnson, B. Batlogg, Z. Benes, J. Fischer, Thermal properties of carbon nanotubes and nanotube-based materials, Appl. Phys. A, 2002, 74, 339-343

[7] N. Behabtu, C. Young, D. Tsentalovich, O. Kleinerman, X. Wang, A. Ma, E. Bengio, R. Hoogerwerf, S. Fairchild, J. Ferguson, B. Maruyama, J. Kono, Y. Talmon, Y. Cohen, M. Otto, M. Pasquali, Strong, light, multifunctional fibers of carbon nanotubes with ultrahigh conductivity, Science, 2013, 339(6116), 182-186 
[8] M. Schulz, Proceedings of the 2014 Nanotechnology Materials and Devices Workshop, University of Cincinnati Nanoworld Lab, Department of Mechanical \& Materials Engineering, 598 Rhodes Hall, Cincinnati, OH 45221-0072

[9] J. Che, T. Cagin, W. Goddard, Thermal conductivity of carbon nanotubes, Nanotechnology, 2000, $11,65-69$

[10] J. Sullivan, Advanced Personal Protection Technology Using Carbon Nanotube Textiles for Firefighters and First Responders, Master Thesis, University of Cincinnati, 2013

[11] Q. Zhang, J. Huang, M. Zhao, W. Qian, F. Wei, Carbon nanotube mass production: Principles and processes, ChemSusChem, 2011, 4(7), 864-889

[12] A. Bhattacharya, J. McGlothlin, Occupational Ergonomics: Theory and Applications, 2nd ed., Boca Raton, Fla., 2012

[13] F. Wang, K. Kuklane, C. Gao, I. Holmér, Can the PHS model (ISO7933) predict reasonable thermophysiological responses while wearing protective clothing in hot environments? Physiol. Meas., 2011, 32(2), 239-249

[14] J. Bugajska, K. Zuzewicz, M. Szmauz-Dybko, M. Konarska, Cardiovascular stress, energy expenditure and subjective perceived ratings of fire fighters during typical fire suppression and rescue tasks, Int. J. Occup. Saf. Ergon., 2007, 13(3), 323-31

[15] D. Cone, D. MacMillan, V. Parwani, Van Gelder C., Threats to life in residential structure fires, Prehosp. Emerg. Care, 2007, 12(3), 297-301

[16] S. Ganguli, S. Sihn, A. Roy, L. Dai, L. Qu, Metalized nanotube tips improve through thickness thermal conductivity in adhesive joints, J. Nanoscience \& Nanotechnology, 2009, 9(3), 1727-1733

[17] F. Incropera, Fundamentals of Heat and Mass Transfer, 7th ed., John Wiley \& Sons, Inc., 2011

[18] J. Pöhls, M. Johnson, M. White, R. Malik, B. Ruff, C. Jayasinghe, M. J. Schulz, V. N. Shanov, Physical properties of carbon nanotube sheets drawn from nanotube arrays, Carbon N. Y., 2012, 50(11), 4175-4183

[19] Safety Components, InterReach Fusion Data Sheet, Tyco Electronics Corporation, P.O. Box 1101, Minneapolis, Minnesota, USA 55440-1101, 2015

[20] DuPont, KEVLAR: Technical Guide; DuPont Advanced Fiber Systems, Customer Inquiry Department, 5401 Jefferson Davis Highway, Richmond, VA 23234, 2015

[21] DuPont, Technical Guide for NOMEX Brand Fiber, H-52720 Revised July, 2001 (Replaces H52703 April, 1999), DuPont Personal Protection, Customer Inquiry Center, 5401 Jefferson Davis Center, Richmond, VA 23234

[22] Crosstech, Black Moisture Barrier - Data Sheet, W. L. Gore \& Associates, Inc., Technical Fabrics Division, 105 Vieve's Way, Elkton, Maryland 21921, 2014

[23] Safety Components, Glide: Thermal Liners - Data Sheet, Lion Products Corporation, 7200 Poe Avenue, Suite 400, Dayton, OH 45414, 2015

[24] R. Lackore, Firefighter Anthropometric Data White Paper, Fire Apparatus Manufacturers Association, FAMA Technical Committee, Chassis Subcommittee, Pierce Manufacturing, October 20, 2007, Firefighter Apparatus Manufacturers Association, P.O. Box 397, Lynnfield, MA 01940-0397

[25] I. Kim, C. Lee, P. Li, B. Corner, S. Paquette, Investigation of air gaps entrapped in protective clothing systems, Fire and Materials, 2002, 26(3), 121-126

[26] American Society of Heating, Refrigerating and Air-Conditioning Engineers (ASHRAE), 2013 ASHRAE Handbook-Fundamentals, ASHRAE Headquarters, 1791 Tullie Circle, N. E. Atlanta, GA 30329

[27] D. Simanovskii, M. Sarkar, A. Irani, C. O'Connell-Rodwell, C. Contag, A. Schwettman, D. Palanker, Cellular tolerance to pulsed heating, SPIE Proceedings, Laser-Tissue Interactions XVI, Vol. 5695, BIOS 2005, 1-6 
[28] B. Cadarette, S. Cheuvront, M. Kolka, L. Stephenson, S. Montain, M. Sawka, Intermittent microclimate cooling during exercise-heat stress in US army chemical protective clothing, Ergonomics, 2006, 49(2), 209-19

[29] H. Pennes, Analysis of tissue and arterial blood temperature in the resting human forearm, J. Appl. Physiol., 1948, 1, 93-122

[30] E. H. Wissler, Mathematical simulation of human thermal behavior using whole-body models, Heat Transf. Med. Biology: Analysis and Applications, Volume 1, Shitzer A. and Eberhart R. C., Eds., Plenum Press, NY, 1985, Chap. 13, 325-373

[31] J. Kluener, V. N. Shanov, C. Jayasinghe, J. Kim, T. Reponen, A. Bhattacharya, B. J. Jetter, A. Schwartz, G. Conroy, K. Simmons, J. T. Jones, R. Koenig, G. Li, M. J. Schulz, Carbon nanotube textiles: New personal protection technology for firefighters and first responders, National Occupational Research Agenda (NORA) Manufacturing Sector Conference: Partnerships to Improve Occupational Safety and Health, Cincinnati, Ohio, September 7-8, 2011

[32] J. Sullivan, M. J. Schulz, K. Vemaganti, A. Bhattacharya, B. J. Jetter, V. N. Shanov, N. Alvarez, J. Kim, A. Schwartz, A. Song, University of Cincinnati Invention Disclosure, Carbon Nanotube Fabric Cooling System, January 2014

[33] W. Li, V. N. Shanov, D. Mast, Y. Song, A. Krishnaswamy, A. Alvarez, M. J. Schulz, D. S. Lashmore, S. N. Yarmolenko, S. Fialkova, S. Desai, University of Cincinnati Invention Disclosure - 115-034, Principles and Practices for Manufacturing Long Nanotubes, August 2014 